\title{
On Cesàro and Copson sequence spaces with weights
}

\author{
S.H. Saker ${ }^{1,2^{*}}$ (D), Maryam M. Abuelwafa ${ }^{2}$, Ahmed M. Zidan ${ }^{3,4}$ and Dumitru Baleanu ${ }^{5,6}$
}

\section{"Correspondence:}

shsaker@gu.edu.eg

${ }^{1}$ Mathematics Division, Faculty of Basic Science, Galala University,

Galala New City 43511, Egypt

Full list of author information is

available at the end of the article

\section{Springer}

\begin{abstract}
In this paper, we prove some properties of weighted Cesàro and Copson sequences spaces by establishing some factorization theorems. The results lead to two-sided norm discrete inequalities with best possible constants and also give conditions for the boundedness of the generalized discrete weighted Hardy and Copson operators.

MSC: 26D20; 46B15; 47J20; 47A63

Keywords: Cesàro sequences space; Copson sequences space; Factorizations; Discrete Hardy's operator; Discrete Copson operator
\end{abstract}

\section{Introduction}

Recently the study of discrete spaces in functional and harmonic analysis has become an active field of research. For example, the study of discrete Cesàro space has been considered by some authors, see for example [6, 22, 27, 29] and the references they cited. Whereas some results from Euclidean functional analysis admit an obvious variant in the discrete setting, some others do not. In fact, it is well known that passage from integral operators to their discrete analogues is not trivial, and each of these two settings requires its own techniques. In this paper, we study the structure of the weighted Cesàro and Copson sequence spaces. Throughout the paper, we assume that $1 \leq p \leq \infty$. The Cesàro function space $\operatorname{Ces}_{p}\left(\mathbb{R}^{+}\right)$is the set of all Lebesgue measurable real functions defined on $\mathbb{R}^{+}=[0, \infty)$ such that

$$
\|f\|_{\operatorname{Ces}_{p}}=\left(\int_{0}^{\infty}\left(\frac{1}{x} \int_{0}^{x}|f(t)| d t\right)^{p} d x\right)^{\frac{1}{p}}<\infty, \quad \text { when } 1 \leq p<\infty
$$

and

$$
\|f\|_{\operatorname{Ces}_{\infty}}=\sup _{x \in \mathbb{R}^{+}, x>0} \frac{1}{x} \int_{0}^{x}|f(t)| d t<\infty, \quad \text { when } p=\infty .
$$

The Cesàro function spaces $\operatorname{Ces}_{p}\left(\mathbb{R}^{+}\right)$for $1 \leq p \leq \infty$ were considered first by Shiue [26] and later by Hassard and Hussein [15], Sy, Zhang, and Lee [28], and Astashkin and Maligranda [4]. They proved that $\operatorname{Ces}_{p}\left(\mathbb{R}^{+}\right)$for $1<p<\infty$ are separable Banach spaces and

(c) The Author(s) 2020. This article is licensed under a Creative Commons Attribution 4.0 International License, which permits use, sharing, adaptation, distribution and reproduction in any medium or format, as long as you give appropriate credit to the original author(s) and the source, provide a link to the Creative Commons licence, and indicate if changes were made. The images or other third party material in this article are included in the article's Creative Commons licence, unless indicated otherwise in a credit line to the material. If material is not included in the article's Creative Commons licence and your intended use is not permitted by statutory regulation or exceeds the permitted use, you will need to obtain permission directly from the copyright holder. To view a copy of this licence, visit http://creativecommons.org/licenses/by/4.0/. 
are not reflexive, and they do not have the fixed point property. They also proved that $\mathrm{Ces}_{\infty}\left(\mathbb{R}^{+}\right)$is a nonseparable Banach space. By the Hardy inequality [14]

$$
\int_{0}^{\infty}\left(\frac{1}{x} \int_{0}^{x}|f(t)| d t\right)^{p} d x \leq\left(\frac{p}{p-1}\right)^{p} \int_{0}^{\infty}|f(x)|^{p} d x
$$

we see that the spaces $L^{p}\left(\mathbb{R}^{+}\right)$(i.e. space of all functions $f$ such that $\left.\left(\int_{0}^{\infty}|f(x)|^{p} d x\right)^{\frac{1}{p}}<\infty\right)$ are continuously embedded into $\operatorname{Ces}_{p}\left(\mathbb{R}^{+}\right)$for $1<p<\infty$ with strict embedding. In other words, $f \in \operatorname{Ces}_{p}\left(\mathbb{R}^{+}\right)$if and only if the operator $T(f)=(1 / x) \int_{0}^{x} f(t) d t$ belongs to $L^{p}\left(\mathbb{R}^{+}\right)$. Also, for $1<p \leq q<\infty$, we see by the inequality

$$
\left(\int_{0}^{\infty} u(x)\left(\int_{0}^{x} f(t) d t\right)^{q} d x\right)^{1 / q} \leq C\left(\int_{0}^{\infty} v(x) f^{p}(x) d x\right)^{1 / p}
$$

due to Opic and Kufner [24] that the spaces $L_{v}^{p}\left(\mathbb{R}^{+}\right)$with weight $v$ are embedded into $\operatorname{Ces}_{q}\left(\mathbb{R}^{+}\right)$with weight $u$ with strict embedding if

$$
\sup _{a<x<\infty}\left(\int_{x}^{\infty} u(t) d t\right)^{1 / q}\left(\int_{a}^{x} v^{1-p^{\prime}}(t) d t\right)^{1 / p^{\prime}}<\infty
$$

It is clear that Hardy's inequality (1) can be interpreted as inclusions between the space of functions $L^{p}\left(\mathbb{R}^{+}\right)$and Cesàro space of functions $\operatorname{Ces}_{p}\left(\mathbb{R}^{+}\right)$; as a consequence, we get that

$$
L^{p} \subseteq \operatorname{Ces}_{p} \quad \text { for } 1<p<\infty .
$$

The Cesàro sequence space $\operatorname{ces}_{p}(\mathbb{N})$ is the set of all real sequences $\left(\lambda_{n}\right)_{n \geq 1}$ on $\mathbb{N}$ that satisfy

$$
\|\lambda\|_{\text {ces } p}=\left(\sum_{n=1}^{\infty}\left(\frac{1}{n} \sum_{k=1}^{n}\left|\lambda_{k}\right|\right)^{p}\right)^{\frac{1}{p}}<\infty, \quad \text { when } 1 \leq p<\infty,
$$

and

$$
\|\lambda\|_{\operatorname{ces} \infty}=\sup _{n \in \mathbb{N}} \frac{1}{n} \sum_{k=1}^{n}\left|\lambda_{k}\right|<\infty, \quad \text { when } p=\infty .
$$

In 1968 the Dutch mathematical society posted a problem of finding an explicit representation of the discrete dual of the Cesàro space of sequences. In [26] Shiue investigated this problem for the first time, and later it was analyzed by Leibowitz [20] and Jagers [16], and for dual spaces of Cesàro space of sequences and functions, we refer to [29]. In particular, they proved that $\operatorname{ces}^{p}(\mathbb{N})$ is a separable reflexive Banach space for $1<p<\infty$, and it does have the fixed point property, and if $1<p<q<\infty$, then $\operatorname{ces}^{p} \subset \operatorname{ces}^{q}$ with continuous strict embedding. The representation result for the dual of the space of sequences of Cesàro type has been extended to the classical Lorentz space of sequences

$$
d(v, q)=\left\{\lambda:\|\lambda\|_{v, q}=\left(\sum_{n=1}^{\infty}\left|\lambda^{*}(n)\right|^{q} v(n)\right)^{1 / q}<\infty\right\},
$$


where $\lambda^{*}(n)$ is the nonincreasing rearrangement of $|\lambda(n)|$ and $q^{*}$ is the conjugate of $q$ by Arińo and Muckenhoupt [3]. They proved that the space $d\left(v^{-q^{*} / q}, q^{*}\right)$ is the dual space of $d(v, q)$ when $v(n)$ is a nonincreasing sequence and satisfies the regularity condition

$$
\frac{1}{n} \sum_{i=1}^{n} v(i) \leq C v(n)
$$

By Hardy's inequality [13],

$$
\sum_{m=1}^{\infty}\left(\frac{1}{m} \sum_{k=1}^{m}\left|\lambda_{k}\right|\right)^{p} \leq\left(\frac{p}{p-1}\right)^{p} \sum_{m=1}^{\infty}\left|\lambda_{m}\right|^{p}, \quad 1<q<\infty,
$$

the space $l^{p}(\mathbb{N})$ (i.e. space of all sequences $\left(\lambda_{n}\right)_{n \geq 1}$ such that $\left.\left(\sum_{n=1}^{\infty}\left|\lambda_{k}\right|^{p}\right)^{\frac{1}{p}}<\infty\right)$ is continuously embedded into $\operatorname{ces}^{p}(\mathbb{N})$ for $1<p<\infty$ with strict embedding. In other words $\lambda_{k} \in \operatorname{ces}^{p}(\mathbb{N})$ if and only if the operator $T(\lambda)=\left(\frac{1}{m} \sum_{k=1}^{m}\left|\lambda_{k}\right|\right)$ belongs to $l^{p}(\mathbb{N})$. Inspired by the development in the continuous case, it is proved in [2, Theorem 4.1] that

$$
\left(\sum_{n=1}^{\infty} u_{n}\left(\sum_{k=1}^{n}\left|a_{k}\right|\right)^{q}\right)^{\frac{1}{q}} \leq C\left(\sum_{n=1}^{\infty} v_{n}\left|a_{n}\right|^{p}\right)^{\frac{1}{p}} \quad \text { for } 1 \leq p \leq q<\infty
$$

holds, where $\left\{u_{n}\right\}_{n=1}^{\infty},\left\{v_{n}\right\}_{n=1}^{\infty}$ are positive sequences if

$$
\sup _{n \in \mathbb{N}}\left(\sum_{k=n}^{\infty} u_{k}\right)^{\frac{1}{q}}\left(\sum_{k=1}^{n} v_{k}^{1-p^{*}}\right)^{\frac{1}{p^{*}}}<\infty, \quad \text { where } p^{*}:=\frac{p}{p-1} .
$$

Inequality (4) can also be interpreted as inclusions between the space of sequences $l_{v}^{p}(\mathbb{N})$ and the Cesàro space of sequences $\operatorname{ces}_{u}^{p}(\mathbb{N})$. In fact this inequality (5) implies that the spaces $l_{v}^{p}(\mathbb{N})$ with weight are embedded into $\operatorname{ces}_{u}^{p}(\mathbb{N})$ with strict embedding; as a consequence, we have that

$$
l_{v}^{p}(\mathbb{N}) \subseteq \operatorname{ces}_{u}^{p}(\mathbb{N}) \quad \text { for } 1<p<\infty
$$

We say that the function $\eta: \mathbb{N} \rightarrow \mathbb{R}$ belongs to the space $l_{\lambda}^{p}(\mathbb{N})$ with a nonnegative weight $\lambda$ defined on $\mathbb{N}=[1, \infty)$ if

$$
\|\eta\|_{l_{\lambda}^{p}(\mathbb{N})}=\left(\sum_{n=1}^{\infty} \lambda(n)|\eta(n)|^{p}\right)^{\frac{1}{p}}<\infty \quad \text { if } 1 \leq p<\infty .
$$

The inclusion interpretation for the discrete spaces has been considered by Bennett in his memorial in [8]. He proved that the validation of inequality (4) is equivalent to an inclusion theorem between the spaces $l^{p}(\mathbb{N})$ and $\operatorname{ces}^{p}(\mathbb{N})$. Precisely Bennett [8] was concerned with the multipliers from $l^{p}(\mathbb{N})$ into $\operatorname{ces}^{p}(\mathbb{N})$, namely those sequences $z=\left\{z_{n}\right\}$ with the property that $y \cdot z \in \operatorname{ces}^{p}(\mathbb{N})$ whenever $y_{n} \in l^{p}(\mathbb{N})$ and $z_{n} \in G(\mathbb{N})$ such that $\sum_{m=1}^{n}\left|z_{m}\right|^{p^{*}}=O(n)$. The set $G(\mathbb{N})$ of all such multipliers clearly satisfies

$$
l^{p}(\mathbb{N}) \cdot G(\mathbb{N}) \subseteq \operatorname{ces}^{p}(\mathbb{N})
$$


Since the discovery of this new way of looking at inequalities, several mathematicians such as Johnson and Mohapatra (see [1, 17-19, 21, 23, 25]) studied the generalizations of the sequence spaces $l^{p}(\mathbb{N})$ and $\operatorname{ces}^{p}(\mathbb{N})$.

In the case of functions, the same factorization results as well as the dual space of Cesàro space are only mentioned in Bennett [8] for the unweighted spaces. A factorization result for the unweighted Cesàro function spaces was proved in Astashkin and Maligranda [5]. In [10] Carton and Heining proved a factorization result which can be considered as a weighted integral analogue of the result obtained by Bennett in [8] for the discrete Hardy operator in the unweighted case. In [7] Barza et al. extended the results proved in [5] and proved some factorization theorems for the Cesàro and Copson functions spaces with weights.

However, the results of Bennett have a big impact in many parts of analysis, but it seems that the corresponding results for weighted spaces are less studied. In some special cases it is possible to translate or adapt almost straightforward the objects and results from the continuous setting to the discrete setting or vice versa; however, in some other cases that is far from being trivial.

In this paper, we develop a new technique to study the structure of weighted Cesàro and Copson sequences spaces and prove some factorization theorems. We mention here that our technique, which is based on some useful lemma proved and designed for this purpose, can be considered as the modification of the technique used in [5] to prove the unweighted results and the technique used in [7] to prove the weighted results for the Cesàro functions space. To the best of the authors' knowledge, the results in this paper for the Cesàro sequences space have not been considered before.

We denote by $\mathcal{H}$ the Cesàro operator and by $\mathcal{M}$ the Copson operator, which are defined by

$$
\mathcal{H} \eta(n):=\frac{1}{n} \sum_{m=1}^{n}|\eta(m)| \text { and } \mathcal{M} \eta(n):=\sum_{s=n}^{\infty} \frac{|\eta(s)|}{s} \text { for } n>0 .
$$

Throughout the paper, the letters $\mathcal{A}, \mathcal{B}, \mathcal{C}, \mathcal{D}$ are used for nonnegative constants independent of the relevant variables that may change from one occurrence to another. In [2] (see also [9]) the authors proved that the discrete inequality

$$
\sum_{m=1}^{\infty} \lambda(m)\left(\frac{1}{m} \sum_{k=1}^{m}|\eta(m)|\right)^{p} \leq C \sum_{m=1}^{\infty} \lambda(m)|\eta(m)|^{p}
$$

holds for all nonnegative sequence $\eta$ and $1<p<\infty$ if

$$
M_{1}:=\sup _{n \in \mathbb{N}}\left(\sum_{k=n}^{\infty} \frac{\lambda(k)}{k^{p}}\right)^{1 / p}\left(\sum_{k=1}^{n} \lambda^{1-q}(k)\right)^{1 / q}<\infty, \quad \text { where } 1 / p+1 / q=1 .
$$

This result proves that the operator $\mathcal{H}$ is bounded on the weighted space $l_{\lambda}^{p}(\mathbb{N})$ if $(7)$ holds. Also in [9] the authors proved that the inequality of Copson type

$$
\sum_{k=1}^{\infty} \lambda(k)\left(\sum_{m=k}^{\infty} \frac{|\eta(m)|}{m}\right)^{p} \leq C \sum_{k=1}^{\infty} \lambda(k)|\eta(k)|^{p}
$$


holds for all nonnegative sequence $\eta$ and $1<p<\infty$ if

$$
M_{2}:=\sup _{n \in \mathbb{N}}\left(\sum_{k=1}^{n} \lambda(k)\right)^{\frac{1}{p}}\left(\sum_{k=n}^{\infty} \frac{\lambda^{1-q}(k)}{k^{q}}\right)^{\frac{1}{q}}<\infty .
$$

Inequality (8) is a generalization of the discrete inequality due to Copson, see [11, 12].

The rest of the paper is divided into three sections: Sect. 2 is devoted to some basic lemmas that will be needed in the proofs of the main results. Section 3 is devoted to the proof of the discrete weighted Cesàro space $\operatorname{Ces}_{\lambda}^{p}(\mathbb{N})$ for $p>1$, and the case when $p=1$, which has not been considered before, is treated separately. In fact we are concerned with the multipliers from $l_{\lambda}^{p}(\mathbb{N})$ into $\operatorname{Ces}_{\lambda}^{p}(\mathbb{N})$, namely those sequences $\beta$ with the property that $\eta \times \beta \in \operatorname{Ces}_{\lambda}^{p}(\mathbb{N})$ whenever $\eta \in l_{\lambda}^{p}(\mathbb{N})$ and $\beta \in A_{\lambda^{(1-q)}}^{q}(\mathbb{N})$. The set $A_{\lambda^{(1-q)}}^{q}(\mathbb{N})$, which will be defined later in the next section, of all such multipliers clearly satisfies

$$
l_{\lambda}^{p}(\mathbb{N}) \cdot A_{\lambda(1-q)}^{q}(\mathbb{N}) \subseteq \operatorname{Ces}_{\lambda}^{p}(\mathbb{N}) .
$$

Section 4 is devoted to the same problem but for the discrete weighted Copson space $\operatorname{Cop}_{\lambda}^{p}(\mathbb{N})$. The results show that the boundedness of the discrete operators $\mathcal{H} \eta$ and $\mathcal{M} \eta$ on the weighted space $l_{\lambda}^{p}(\mathbb{N})$ can be obtained from general norm discrete inequalities.

\section{Basic lemmas}

In this section, we state and prove the main basic lemmas that are needed in the rest of the papers. The first two are adapted from [9].

Lemma 2.1 Let $0<\gamma \leq 1$, then

$$
\left(\sum_{m=1}^{n} \phi(m)\right)^{\gamma} \geq \gamma \sum_{m=1}^{n} \phi(m)\left(\sum_{s=1}^{m} \phi(s)\right)^{\gamma-1}
$$

Lemma 2.2 Let $0<\gamma \leq 1$, then

$$
\left(\sum_{m=n}^{\infty} \phi(m)\right)^{\gamma} \geq \gamma \sum_{m=n}^{\infty} \phi(m)\left(\sum_{s=m}^{\infty} \phi(s)\right)^{\gamma-1} .
$$

In what follows, it will be convenient to use the convention $\sum_{m=a}^{b} y(m)=0$, whenever $a>b$,

$$
\Delta\left(\sum_{s=k_{0}}^{n-1} y(s)\right)=y(n), \quad \text { and } \quad\left(\sum_{s=m}^{n-1} \Delta y(s)\right)=y(n)-y(s) .
$$

The following lemmas will be also needed in the proofs.

Lemma 2.3 Let $\phi, \varphi, h$ be nonnegative sequences. If

$$
\sum_{k=1}^{n} \phi(k) \leq \sum_{k=1}^{n} \varphi(k)
$$


then

$$
\sum_{k=1}^{N} \phi(k) H(k) \leq \sum_{k=1}^{N} \varphi(k) H(k), \quad \text { where } H(n)=\sum_{k=n}^{N} h(k) \text {. }
$$

Proof By defining $F(n)=\sum_{k=1}^{n} \phi(k)$ and $G(n)=\sum_{k=1}^{n} \varphi(k)$, we see from (12) that $F(n) \leq$ $G(n)$. Applying summation by parts

$$
\sum_{k=1}^{N} \Delta \eta(k) v(k+1)=\left.\eta(k) v(k)\right|_{k=1} ^{N+1}-\sum_{k=1}^{N} \eta(k) \Delta v(k)
$$

on the left-hand side of (13), we get

$$
\sum_{k=1}^{N} \phi(k) H(k)=\left.F(k) H(k)\right|_{k=1} ^{N}+\sum_{k=1}^{N} F(k+1) h(k) .
$$

By noting that $F(1)=H(N+1)=0$ and making use of $F(k+1) \leq G(k+1)$, we get that

$$
\sum_{k=1}^{N} \phi(k) H(k) \leq \sum_{k=1}^{N} G(k+1) h(k)
$$

Applying summation by parts on the right-hand side of inequality (15), we have that

$$
\sum_{k=1}^{N} G(k+1) h(k)=\left.G(k) H(k)\right|_{k=1} ^{N}+\sum_{k=1}^{N} \varphi(k) H(k) .
$$

Since $G(1)=H(N)=0$, we have from the last inequality that

$$
\sum_{k=1}^{N} G(k+1) h(k) \leq \sum_{k=1}^{N} \varphi(k) H(k)
$$

Combining (15) and (16), we get

$$
\sum_{k=1}^{N} \phi(k) H(k) \leq \sum_{k=1}^{N} \varphi(k) H(k)
$$

which is the desired inequality (13). The proof is complete.

Lemma 2.4 Let $\phi, \varphi, h$ be nonnegative sequences. If $C$ is a positive constant such that

$$
F(n)=\sum_{k=n}^{N} \phi(k) \leq C \sum_{k=n}^{N} \varphi(k)=C G(n)
$$

then

$$
\sum_{k=1}^{N} \phi(k) H(k) \leq C \sum_{k=1}^{N} \varphi(k) H(k), \quad \text { where } H(n)=\sum_{k=1}^{n} h(k) \text {. }
$$


Proof Applying summation by parts twice.

Lemma 2.5 Let $\varphi, \psi$ be nonnegative sequences, then

$$
\sum_{k=1}^{N-1} \varphi(k)\left(\sum_{s=k}^{N-1} \psi(s)\right)=\sum_{k=1}^{N-1} \psi(k)\left(\sum_{s=1}^{k} \varphi(s)\right)
$$

Proof By defining $\Psi(k)=\sum_{s=k}^{N-1} \psi(s)$ and applying summation by parts on the left-hand side of (17) with $\eta(k)=\Psi(k)$ and $\Delta v(k)=\varphi(k)$, we get

$$
\begin{aligned}
\sum_{k=1}^{N-1} \varphi(k)\left(\sum_{s=k}^{N-1} \psi(s)\right) & =\sum_{k=1}^{N-1} \varphi(k) \Psi(k) \\
& =\left.\Psi(k) v(k)\right|_{k=1} ^{N}-\sum_{k=1}^{N-1} \Delta \Psi(k) v(k+1),
\end{aligned}
$$

where $v(k)=\sum_{s=1}^{k-1} \varphi(s)$. Since $v(1)=\Psi(N)=0$, we have

$$
\sum_{k=1}^{N-1} \varphi(k)\left(\sum_{s=k}^{N-1} \psi(s)\right)=\sum_{k=1}^{N-1}(-\Delta \Psi)(k) v(k+1)=\sum_{k=1}^{N-1} \psi(k)\left(\sum_{s=1}^{k} \varphi(s)\right) .
$$

The proof is complete.

\section{Weighted Cesàro sequences space}

In this section, we prove a factorization theorem of the discrete weighted Cesàro space $\operatorname{Ces}_{\lambda}^{p}(\mathbb{N})$; as a consequence, we recover some best known forms of the discrete Hardy type inequalities as special cases. We start by presenting the basic definitions.

Definition 3.1 The discrete weighted Cesàro space $\operatorname{Ces}_{\lambda}^{p}(\mathbb{N})$ for $p \geq 1$ with a discrete weight $\lambda$ is the space of all sequences $\eta$ defined on $\mathbb{N}$ with a norm

$$
\|\eta\|_{\operatorname{Ces}_{\lambda}^{p}(\mathbb{N})}=\left(\sum_{n=1}^{\infty} \lambda(n)\left(\frac{1}{n} \sum_{m=1}^{n}|\eta(m)|\right)^{p}\right)^{1 / p}<\infty .
$$

Claim 1 The space $\operatorname{Ces}_{\lambda}^{p}(\mathbb{N})$ with norm (18) for $p>1$ is obviously a Banach space, and if the discrete weight $\lambda$ satisfies (7), then the operator $\mathcal{H}$ is bounded on $l_{\lambda}^{p}(\mathbb{N})$, and we have $l_{\lambda}^{p}(\mathbb{N}) \subseteq \operatorname{Ces}_{\lambda}^{p}(\mathbb{N})$.

Definition 3.2 We define the sequence space $A_{\lambda}^{p}(\mathbb{N})$ for $p \geq 1$ of positive sequences $\beta$ of real numbers defined on $\mathbb{N}$ with a discrete weight $\lambda$ by

$$
A_{\lambda}^{p}(\mathbb{N})=\left\{\beta: \sum_{m=1}^{n}|\beta(m)|^{p} \lambda(m)=O(\Lambda(n))\right\}, \quad \text { where } \Lambda(n)=\sum_{m=1}^{n} \lambda(m),
$$

with the norm

$$
\|\beta\|_{A_{\lambda}^{p}(\mathbb{N})}=\sup _{n>0}\left(\frac{1}{\Lambda(n)} \sum_{m=1}^{n}|\beta(m)|^{p} \lambda(m)\right)^{1 / p}<\infty .
$$


We denote

$$
\mathcal{J}:=\inf \left\{\|\eta\|_{l_{\lambda}^{p}(\mathbb{N})}\|\beta\|_{A_{\lambda(1-q)}^{q}(\mathbb{N})}\right\}
$$

The infimum is taken over all possible decompositions of $\hbar=\eta \times \beta$ with $\eta \in l_{\lambda}^{p}(\mathbb{N})$ and $\beta \in A_{\lambda(1-q)}^{q}(\mathbb{N})$.

Definition 3.3 We denote by $\mathcal{M}^{*}$ the discrete class of weights $\lambda$ such that, for all $n \geq 1$, it follows that

$$
\left(\sum_{k=n}^{\infty} \frac{\lambda(k)}{k^{p}}\right)^{1 / p}\left(\sum_{k=1}^{n} \lambda^{1-q}(k)\right)^{1 / q} \leq \mathcal{A}^{*}
$$

for $p>1$, where $1 / p+1 / q=1$ and $\mathcal{A}^{*}$ is the smallest positive constant such that (21) holds.

Definition 3.4 We denote by $\mathcal{M}_{*}$ the discrete class of weights such that, for all $n \geq 1$, it follows that the reverse of inequality (21) holds, and we denote by $\mathcal{A}_{*}$ the largest positive constant for which this reverse holds.

Definition 3.5 The weight $\lambda$ belongs to the class $\mathcal{M}$ if it belongs to $\mathcal{M}^{*}$ and $\mathcal{M}_{*}$.

Theorem 3.1 Assume that $p>1$ and $\lambda \in \mathcal{M}$. Then $\hbar \in \operatorname{Ces}_{\lambda}^{p}(\mathbb{N})$ iff it admits a factorization $\hbar=\eta \times \beta$ with $\eta \in l_{\lambda}^{p}(\mathbb{N})$ and $\beta \in A_{\left(\lambda^{1-q}\right)}^{q}(\mathbb{N})$. Moreover,

$$
\mathcal{J} \mathcal{A}_{*} \leq\|\hbar\|_{\operatorname{Ces}_{\lambda}^{p}(\mathbb{N})} \leq q^{1 / q} p^{1 / p} \mathcal{J} \mathcal{A}^{*}
$$

Proof We start by proving the imbedding $\hookrightarrow$ i.e. we prove that the sequence $\hbar=\eta \times \beta \in$ $\operatorname{Ces}_{\lambda}^{p}(\mathbb{N})$, where $\eta \in l_{\lambda}^{p}(\mathbb{N})$ and $\beta \in A_{\left(\lambda^{1-q}\right)}^{q}(\mathbb{N})$. By defining $w(n)=\left(\sum_{m=1}^{n} \lambda^{1-q}(m)\right)^{-1 / p q}$ and employing discrete Hölder's inequality

$$
\sum|\eta(n)||\beta(n)| \leq\left[\sum|\eta(n)|^{q}\right]^{1 / q}\left[\sum^{p}|\beta(n)|^{p}\right]^{1 / p}
$$

with $1 / p+1 / q=1$, we get that

$$
\begin{aligned}
\sum_{m=1}^{n}|\hbar(m)| & =\sum_{m=1}^{n}|\eta(m) \beta(m)| \\
& \leq\left(\sum_{m=1}^{n}|\eta(m)|^{p} \frac{\lambda(m)}{w^{p}(m)}\right)^{1 / p}\left(\sum_{m=1}^{n}|\beta(m)|^{q} \lambda^{1-q}(m) w^{q}(m)\right)^{1 / q} .
\end{aligned}
$$

Now, Definition (19) leads to

$$
\left(\sum_{m=1}^{n}|\beta(m)|^{q} \lambda^{1-q}(m)\right)^{1 / q} \leq\|\beta\|_{A_{(\lambda-q)}^{q}}\left(\sum_{m=1}^{n} \lambda^{1-q}(m)\right)^{1 / q} .
$$


Saker et al. Journal of Inequalities and Applications

(2021) $2021: 1$

Page 9 of 23

By applying Lemma 2.3 , since $w^{q}(n)$ is decreasing, we obtain that

$$
\left(\sum_{m=1}^{n}|\beta(m)|^{q} \lambda^{1-q}(m) w^{q}(m)\right)^{1 / q} \leq\|\beta\|_{A_{(\lambda 1-q)}^{q}(\mathbb{N})}\left(\sum_{m=1}^{n} \lambda^{1-q}(m) w^{q}(m)\right)^{1 / q} .
$$

Dividing (24) by $n>0$ and summing from 1 to $\infty$ and then using (25) and applying Lemma 2.5, we get that

$$
\begin{aligned}
& \sum_{n=1}^{\infty}\left(\frac{1}{n} \sum_{m=1}^{n}|\hbar(m)|\right)^{p} \lambda(n) \\
& \quad \leq\|\beta\|_{A_{\left(\lambda^{1-q)}\right.}^{q}(\mathbb{N})}^{p} \sum_{n=1}^{\infty}\left(\sum_{m=1}^{n}|\eta(m)|^{p} \frac{\lambda(m)}{w^{p}(m)}\right)\left(\sum_{s=1}^{n} \lambda^{1-q}(s) w^{q}(s)\right)^{p-1} \frac{\lambda(n)}{n^{p}} \\
& \quad=\|\beta\|_{A_{(\lambda 1-q)}^{q}(\mathbb{N})}^{p} \sum_{n=1}^{\infty}|\eta(n)|^{p} \frac{\lambda(n)}{w^{p}(n)}\left(\sum_{s=n}^{\infty} \frac{\lambda(s)}{s^{p}}\left(\sum_{\tau=1}^{s} \lambda^{1-q}(\tau) w^{q}(\tau)\right)^{p-1}\right) .
\end{aligned}
$$

By employing (10) with $\phi(x)=\lambda^{1-q}(x)$ and $\gamma=1 / q<1$ and using the definition of $w(n)$, we obtain that

$$
\sum_{m=1}^{n} \lambda^{1-q}(m)\left(\sum_{s=1}^{m} \lambda^{1-q}(s)\right)^{1 / q-1} \leq q\left(\sum_{m=1}^{n} \lambda^{1-q}(m)\right)^{1 / q}
$$

and then

$$
\begin{aligned}
\sum_{n=1}^{\infty}( & \left.\frac{1}{n} \sum_{m=1}^{n}|\hbar(m)|\right)^{p} \lambda(n) \\
\leq & q^{p-1}\|\beta\|_{A_{(\lambda 1-q)}^{q}(\mathbb{N})}^{p} \sum_{n=1}^{\infty}|\eta(n)|^{p} \lambda(n)\left(\sum_{m=1}^{n} \lambda^{1-q}(m)\right)^{1 / q} \sum_{s=n}^{\infty} \frac{\lambda(s)}{s^{p}}\left(\sum_{\tau=1}^{s} \lambda^{1-q}(\tau)\right)^{(p-1) / q} \\
= & R \sum_{n=1}^{\infty}|\eta(n)|^{p} \lambda(n)\left(\sum_{m=1}^{n} \lambda^{1-q}(m)\right)^{1 / q} \sum_{s=n}^{\infty} \frac{\lambda(s)}{s^{p}}\left(\sum_{x=s}^{\infty} \frac{\lambda(x)}{x^{p}}\right)^{\frac{(1-p)}{p}} \\
& \times\left[\left(\sum_{\tau=1}^{s} \lambda^{1-q}(\tau)\right)^{(p-1) / q}\left(\sum_{x=s}^{\infty} \frac{\lambda(x)}{x^{p}}\right)^{(p-1) / p}\right]
\end{aligned}
$$

where $R=q^{p-1}\|\beta\|_{A_{(\lambda 1-q)}^{q}}^{p}(\mathbb{N})$. By using the definition of $\mathcal{A}^{*}$ and employing (11) with $\gamma=$ $1 / p<1$ and $\phi=\lambda(n) / n^{p}$, we get that

$$
\begin{aligned}
\sum_{n=1}^{\infty}( & \left.\frac{1}{n} \sum_{m=1}^{n}|\hbar(m)|\right)^{p} \lambda(n) \\
\leq & q^{p-1}\left(\mathcal{A}^{*}\right)^{p-1}\|\beta\|_{A_{\left(\lambda^{1-q}\right)}^{p}}^{(\mathbb{N})} \sum_{n=1}^{\infty}|\eta(n)|^{p} \lambda(n) \\
& \times\left(\sum_{m=1}^{n} \lambda^{1-q}(m)\right)^{1 / q} \sum_{s=n}^{\infty} \frac{\lambda(s)}{s^{p}}\left(\sum_{x=s}^{\infty} \frac{\lambda(x)}{x^{p}}\right)^{-1 / q}
\end{aligned}
$$




$$
\begin{aligned}
& \leq p R\left(\mathcal{A}^{*}\right)^{p-1} \sum_{n=1}^{\infty}|\eta(n)|^{p} \lambda(n)\left(\sum_{m=1}^{n} \lambda^{1-q}(m)\right)^{1 / q}\left(\sum_{s=n}^{\infty} \frac{\lambda(s)}{s^{p}}\right)^{1 / p} \\
& \leq p\left(\mathcal{A}^{*}\right)^{p} R \sum_{n=1}^{\infty}|\eta(n)|^{p} \lambda(n) \leq p\left(\mathcal{A}^{*}\right)^{p} R\|\eta\|_{l_{\lambda}^{p}(\mathbb{N})}^{p}
\end{aligned}
$$

This implies that $\hbar \in \operatorname{Ces}_{\lambda}^{p}(\mathbb{N})$ and

$$
\|\hbar\|_{\operatorname{Ces}_{\lambda}^{p}(\mathbb{N})} \leq q^{1 / q} p^{1 / p} \mathcal{A}^{*} \inf \left\{\|\eta\|_{l_{\lambda}^{p}(\mathbb{N})}\|\beta\|_{A_{(\lambda 1-q)}^{q}(\mathbb{N})}\right\}
$$

and the infimum is determined over all possible factorization of $\hbar$. This leads to the completeness of the first part of the proof. For the reversed direction $\hookleftarrow$, we prove that $\operatorname{Ces}_{\lambda}^{p}(\mathbb{N}) \subseteq l_{\lambda}^{p}(\mathbb{N}) \times A_{\left(\lambda^{1-q}\right)}^{q}(\mathbb{N})$. Now let $\hbar \in \operatorname{Ces}_{\lambda}^{p}(\mathbb{N})$ and define

$$
v(n):=\frac{1}{\lambda(n)} \sum_{s=n}^{\infty} \frac{\lambda(s)}{s}\left(\frac{1}{s} \sum_{m=1}^{s}|\hbar(m)|\right)^{p-1}>0 \text { for } n>0,
$$

and let

$$
\eta(n)=|\hbar(n)|^{1 / p} v^{1 / p}(n), \quad \text { and } \quad \beta(n)=|\hbar(n)|^{1 / q} v^{-1 / p}(n) .
$$

Since $\eta^{p}(n)=|\hbar(n)| v(n)$, then we have

$$
\|\eta\|_{l_{\lambda}^{p}(\mathbb{N})}^{p}=\sum_{m=1}^{\infty} \eta^{p}(m) \lambda(m)=\sum_{m=1}^{\infty}|\hbar(m)| \sum_{s=m}^{\infty} \frac{\lambda(s)}{s}\left(\frac{1}{s} \sum_{m=1}^{s}|\hbar(m)|\right)^{p-1} .
$$

By applying Lemma 2.5 , we obtain that

$$
\begin{aligned}
\|\eta\|_{l_{\lambda}^{p}(\mathbb{N})}^{p} & =\sum_{m=1}^{\infty}\left(\sum_{s=1}^{m}|\hbar(s)| \Delta s\right)\left(\frac{1}{m} \sum_{k=1}^{m}|\hbar(k)|\right)^{p-1} \frac{\lambda(m)}{m} \\
& =\sum_{m=1}^{\infty}\left(\frac{1}{m} \sum_{k=1}^{m}|\hbar(k)|\right)\left(\frac{1}{m} \sum_{k=1}^{m}|\hbar(k)|\right)^{p-1} \lambda(m) \\
& =\sum_{m=1}^{\infty} \lambda(m)\left(\frac{1}{m} \sum_{k=1}^{m}|\hbar(k)|\right)^{p}=\|\hbar\|_{\operatorname{Ces}_{\lambda}^{p}(\mathbb{N})}^{p}
\end{aligned}
$$

i.e. $\|\eta\|_{l_{\lambda}^{p}(\mathbb{N})}=\|\hbar\|_{\operatorname{Ces}_{\lambda}^{p}(\mathbb{N})}<\infty$. By Hölder's inequality, we see that

$$
\begin{aligned}
\left(\sum_{m=1}^{n} \beta^{q}(m) \lambda^{1-q}(m)\right)^{p} & =\left(\sum_{m=1}^{n}|\hbar(m)| v^{-q / p}(m) \lambda^{1-q}(m)\right)^{p} \\
& \leq\left(\sum_{m=1}^{n}|\hbar(m)|\right)^{p-1}\left(\sum_{m=1}^{n}|\hbar(m)| v^{-q}(m) \lambda^{-q}(m)\right) .
\end{aligned}
$$


Now (26) implies that $\Delta[\lambda(n) v(n)] \leq 0$ and then $\lambda(n) v(n)$ is decreasing. Multiplying (28) by $\sum_{s=n}^{\infty} s^{-p} \lambda(s)$ and using the fact that $\lambda(n) v(n)$ is decreasing, we get that

$$
\begin{aligned}
& \left(\sum_{s=n}^{\infty} \frac{\lambda(s)}{s^{p}}\right)\left(\sum_{m=1}^{n} \beta^{q}(m) \lambda^{1-q}(m)\right)^{p} \\
& \leq\left(\sum_{s=n}^{\infty} \frac{\lambda(s)}{s^{p}}\left(\sum_{m=1}^{s}|\hbar(m)|\right)^{p-1}\right)\left(\sum_{m=1}^{n} \frac{|\hbar(m)|}{\lambda q(m)} v^{-q}(m)\right) \\
& =\lambda(n) v(n) \sum_{m=1}^{n} \frac{|\hbar(m)|}{\lambda^{q}(m)} v^{-q}(m) \leq \sum_{m=1}^{n}|\hbar(m)| v^{1-q}(m) \lambda^{1-q}(m) \\
& =\sum_{m=1}^{n}|\hbar(m)| v^{1-q}(m) \lambda^{1-q}(m)=\sum_{m=1}^{n} \beta^{q}(m) \lambda^{1-q}(m) .
\end{aligned}
$$

Since $|\beta(n)|^{q}=|\hbar(n)| v^{1-q}(n)$, we obtain

$$
\left(\frac{\sum_{m=1}^{n} \beta^{q}(m) \lambda^{1-q}(m)}{\sum_{m=1}^{n} \lambda^{1-q}(m)}\right)^{1 / q} \leq\left(\sum_{s=n}^{\infty} \frac{\lambda(s)}{s^{p}}\right)^{-1 / p}\left(\sum_{m=1}^{n} \lambda^{1-q}(m)\right)^{-1 / q} .
$$

Hence

$$
\left(\frac{1}{\sum_{m=1}^{n} \lambda^{1-q}(m)} \sum_{m=1}^{n} \beta^{q}(m) \lambda^{1-q}(m)\right)^{1 / q} \leq \frac{1}{\mathcal{A}_{*}},
$$

which shows that $\beta$ belongs to $A_{\left(\lambda^{1-q)}\right.}^{q}(\mathbb{N})$, and

$$
\|\hbar\|_{\operatorname{Ces}_{\lambda}^{p}(\mathbb{N})}=\|\eta\|_{l_{\lambda}^{p}(\mathbb{N})} \geq \mathcal{A}_{*}\|\eta\|_{l_{\lambda}^{p}(\mathbb{N})}\|\beta\|_{A_{(\lambda 1-q)}^{q}(\mathbb{N})}
$$

This implies that

$$
\|\hbar\|_{\operatorname{Ces}_{\lambda}^{p}(\mathbb{N})} \geq \mathcal{A}_{*} \inf \left\{\|\eta\|_{l_{\lambda}^{p}(\mathbb{N})}\|\beta\|_{A_{(\lambda-q)}^{q}(\mathbb{N})}\right\}
$$

which gives the left-hand side of inequality (22), and the infimum is taken over all possible decompositions of $\hbar=\eta \cdot \beta$ such that $\eta \in l_{\lambda}^{p}(\mathbb{N})$ and $\beta \in A_{\left(\lambda^{1-q}\right)}^{q}(\mathbb{N})$. The proof is complete.

Example 1 If we take $\beta(n)=1$, then

$$
\|\hbar\|_{\operatorname{Ces}_{\lambda}^{p}(\mathbb{N})}=\|\mathcal{H} \eta\|_{l_{\lambda}^{p}(\mathbb{N})}=\left(\sum_{n=1}^{\infty} \lambda(n)\left(\frac{1}{n} \sum_{m=1}^{n}|\eta(m)|\right)^{p}\right)^{1 / p},
$$

where $\mathcal{H} \eta(n)=(1 / n) \sum_{m=1}^{n}|\eta(m)|$ is the Hardy operator. Then we have from Theorem 3.1 that the Hardy operator is bounded on $l_{\lambda}^{p}(\mathbb{N})$ if and only if

$$
\left(\sum_{k=n}^{\infty} \frac{\lambda(k)}{k^{p}}\right)^{1 / p}\left(\sum_{k=1}^{n} \lambda^{1-q}(k)\right)^{1 / q} \leq \mathcal{C}
$$


Moreover,

$$
\mathcal{A}_{*}\|\eta(n)\|_{l_{\lambda}^{p}(\mathbb{N})} \leq\|\mathcal{H} \eta(n)\|_{l_{\lambda}^{p}(\mathbb{N})} \leq q^{1 / q} p^{1 / p} \mathcal{A}^{*}\|\eta(n)\|_{l_{\lambda}^{p}(\mathbb{N})}{ }^{\prime}
$$

where $\mathcal{A}_{*}$ and $\mathcal{A}^{*}$ are defined as above.

Now, we consider the case when $p=1$, which is an independent and important case in its own. In this case, we consider the space $\operatorname{Ces}_{\lambda}^{1}(\mathbb{N})$ which will be obtained from $\operatorname{Ces}_{\lambda}^{p}(\mathbb{N})$ by putting $p=1$. On the other hand, if we consider $\|\beta\|_{\infty}=\sup _{n>0}|\beta(n)|<\infty$, we see that

$$
\frac{1}{\Lambda(n)} \sum_{m=1}^{n}|\beta(m)| \lambda(m) \leq\|\beta\|_{\infty} \frac{1}{\Lambda(n)} \sum_{m=1}^{n} \lambda(m)=\|\beta\|_{\infty} .
$$

This allows us to replace the space $A_{\lambda}^{1}(\mathbb{N})$ with the space $l^{\infty}(\mathbb{N})$ with a norm $\|\beta\|_{\infty}=$ ess $\sup _{n>0}|\beta(n)|<\infty$. Now, we consider a new space $\mathcal{U}$ of weights which is defined by

$$
\mathcal{U}:=\left\{\lambda: \frac{1}{\lambda(n)} \sum_{s=n}^{\infty} \frac{\lambda(s)}{s} \leq C \text { for } n>0\right\},
$$

and assume that there exists a positive constant $\mathcal{P}^{*}$ which is the smallest constant such that the inequality

$$
\frac{1}{\lambda(n)} \sum_{s=n}^{\infty} \frac{\lambda(s)}{s} \leq C
$$

holds, and there exists a positive constant $\mathcal{P}_{*}$ which is the largest constant for which the reverse of (30) holds. In the following, we denote

$$
\mathcal{F}=\inf \left\{\|\eta\|_{l_{\lambda}^{1}(\mathbb{N})}\|\beta\|_{\infty}\right\}
$$

where the infimum is taken over all possible decompositions of $\hbar=\eta \times \beta$ with $\eta \in l_{\lambda}^{1}(\mathbb{N})$ and $\beta \in l^{\infty}(\mathbb{N})$.

Theorem 3.2 Let $\lambda$ belong to the class $\mathcal{U}$. Then $\hbar \in \operatorname{Ces}_{\lambda}^{1}(\mathbb{N})$ iff it can be represented by the factorization $\hbar=\eta \times \beta$, with $\eta \in l_{\lambda}^{1}(\mathbb{N})$ and $\beta \in l^{\infty}(\mathbb{N})$. Moreover,

$$
\mathcal{F} \mathcal{P}_{*} \leq\|\hbar\|_{\operatorname{Ces}_{\lambda}^{1}(\mathbb{N})} \leq \mathcal{F} \mathcal{P}^{*}
$$

Proof Let $\eta \in l_{\lambda}^{1}(\mathbb{N})$ and $\beta \in l^{\infty}(\mathbb{N})$. We prove that the function $\hbar=\eta \times \beta \in \operatorname{Ces}_{\lambda}^{1}(\mathbb{N})$. By employing Hölder's inequality, we get

$$
\sum_{n=1}^{\infty}\left(\frac{1}{n} \sum_{m=1}^{n}|\hbar(m)|\right) \lambda(n) \leq\|\beta\|_{\infty} \sum_{n=1}^{\infty}\left(\frac{1}{n} \sum_{m=1}^{n}|\eta(m)|\right) \lambda(n),
$$

which implies that $\hbar \in \operatorname{Ces}_{\lambda}^{1}(\mathbb{N})$. Also since $l_{\lambda}^{1}(\mathbb{N}) \times l^{\infty}(\mathbb{N})=l_{\lambda}^{1}(\mathbb{N})$, then $\operatorname{Ces}_{\lambda}^{1}(\mathbb{N})=l_{\lambda}^{1}(\mathbb{N}) \times$ $l^{\infty}(\mathbb{N})=l_{\lambda}^{1}(\mathbb{N})$. Now, we prove the right-hand side of inequality (31). By Lemma 2.5 and 
taking into account that $\lambda$ belongs to the class $\mathcal{U}$, we have that

$$
\|\hbar\|_{\operatorname{Ces}_{\lambda}^{1}(\mathbb{N})} \leq \mathcal{P}^{*}\|\eta\|_{l_{\lambda}^{1}}\|\beta\|_{\infty}
$$

This completes the first part of the proof and the right-hand side of (31). Conversely, let $\hbar \in \operatorname{Ces}_{\lambda}^{1}(\mathbb{N})$ and

$$
v(n):=\frac{1}{\lambda(n)} \sum_{s=n}^{\infty} \frac{\lambda(s)}{s}>0 \quad \text { for } n>0,
$$

and set

$$
\eta(n)=|\hbar(n)| v(n) \operatorname{sign} \hbar(n) \quad \text { and } \quad \beta(n)=1 / v(n) .
$$

It is easy to see that $\|\eta\|_{l^{1}(\lambda)}=\|\hbar\|_{\operatorname{Ces}_{\lambda}^{1}(\mathbb{N})}<\infty$. Since $\lambda$ belongs to the class $\mathcal{U}$ and

$$
\beta \in l^{\infty}(\mathbb{N}) \quad \text { with }\|\beta\|_{\infty} \leq 1 / \mathcal{P}_{*} \text {, }
$$

we have that

$$
\|\hbar\|_{\operatorname{Ces}_{\lambda}^{1}(\mathbb{N})}=\|\eta\|_{l_{\lambda}^{1}(\mathbb{N})} \geq \mathcal{P}_{*}\|\eta\|_{l_{\lambda}^{1}(\mathbb{N})}\|\beta\|_{\infty}
$$

and then we get the left-hand side of inequality (31). This completes the proof.

Remark 3.1 Theorem 3.2 proves that the Hardy operator $\mathcal{H} \eta(n)=\frac{1}{n} \sum_{m=1}^{n}|\eta(m)|$ for $n>0$ is bounded on $l_{\lambda}^{1}(\mathbb{N})$ if and only if there exists $C>0$ such that

$$
\frac{1}{\lambda(n)} \sum_{s=n}^{\infty} \frac{\lambda(s)}{s} \leq C
$$

In the following, we denote by $l_{n^{\alpha}}^{p}(\mathbb{N})$, $\operatorname{Ces}_{n^{\alpha}}^{p}(\mathbb{N})$, and $A_{n^{\alpha}}^{p}(\mathbb{N})$ the spaces with the power weight $\lambda(n)=n^{\alpha}$. In analogy with the general case, we denote

$$
\mathcal{S}:=\inf \left\{\|\eta\|_{l_{n^{\alpha}}^{p}(\mathbb{N})}\|\beta\|_{A_{n^{\alpha(1-q)}}^{q}(\mathbb{N})}\right\}
$$

where the infimum is taken over all possible decompositions of $\hbar=\eta \times \beta$ with $\eta \in l_{n^{\alpha}}^{p}(\mathbb{N})$ and $\beta \in A_{n^{\alpha(1-q)}}^{q}(\mathbb{N})$. This gives us the following result.

Corollary 3.1 Assume that $p>1$ and $p-1>\alpha>0$. Then $\hbar \in \operatorname{Ces}_{n^{\alpha}}^{p}(\mathbb{N})$ iff $\hbar$ can be factorized into two sequences $\eta \in l_{n^{\alpha}}^{p}(\mathbb{N})$ and $\beta \in A_{n^{\alpha(1-q)}}^{q}(\mathbb{N})$ such $\hbar=\eta \times \beta$. Moreover,

$$
\left(\frac{1}{p}\right)^{1 / p}\left(\frac{1}{q}\right)^{1 / q} \frac{p \mathcal{S}}{(p-\alpha-1)} \leq\|\hbar\|_{\operatorname{Ces}_{\alpha}^{p}(\mathbb{N})} \leq \frac{2 p \mathcal{S}}{(p-\alpha-1)} .
$$

Proof By using $\lambda(n)=n^{\alpha}$, we see that condition (21) now reads

$$
\left(\sum_{m=n}^{\infty} m^{\alpha-p}\right)^{1 / p}\left(\sum_{m=1}^{n} m^{\alpha(1-q)}\right)^{1 / q} .
$$


By employing the inequality [14]

$$
\gamma z^{\gamma-1}(y-z) \leq y^{\gamma}-z^{\gamma} \leq \gamma y^{\gamma-1}(y-z) \text { for } y \geq z>0, \gamma \geq 1 \text {, or } \gamma<0 \text {, }
$$

with $\gamma=\alpha-p+1<0$, we obtain

$$
\begin{aligned}
\Delta\left(k^{\alpha-p+1}\right) & =(k+1)^{\alpha-p+1}-k^{\alpha-p+1} \geq(\alpha-p+1) k^{\alpha-p}[(k+1)-k] \\
& =(\alpha-p+1) k^{\alpha-p} .
\end{aligned}
$$

For $0<\gamma=\alpha(1-q)+1<1$, we also have by using the inequality [14]

$$
\gamma y^{\gamma-1}(y-z) \leq y^{\gamma}-z^{\gamma} \leq \gamma z^{\gamma-1}(y-z) \quad \text { for } y \geq z>0 \text { and } 0<\gamma<1
$$

that

$$
\begin{aligned}
\Delta\left(s^{\alpha(1-q)+1}\right) & =(s+1)^{\alpha(1-q)+1}-s^{\alpha(1-q)+1} \\
& \leq(\alpha(1-q)+1) s^{\alpha(1-q)}[(s+1)-s] \\
& =(\alpha(1-q)+1) s^{\alpha(1-q)} .
\end{aligned}
$$

Then, from (36) and (38) in (34), we get that

$$
\begin{aligned}
& \left(\sum_{m=n}^{\infty} m^{\alpha-p}\right)^{1 / p}\left(\sum_{m=1}^{n} m^{\alpha(1-q)}\right)^{1 / q} \\
& \geq\left(\frac{\sum_{m=n}^{\infty} \Delta\left(m^{\alpha-p+1}\right)}{(\alpha-p+1)}\right)^{1 / p}\left(\frac{\sum_{m=1}^{n} \Delta\left(m^{\alpha(1-q)+1}\right)}{(\alpha(1-q)+1)}\right)^{1 / q} \\
& =\left(\frac{-n^{\frac{\alpha+1}{p}-1}}{(\alpha-p+1)^{1 / p}}\right)\left(\frac{n^{\frac{\alpha}{q}(1-q)+\frac{1}{q}}}{(\alpha(1-q)+1)^{1 / q}}\right) \\
& =\frac{1}{p-\alpha-1}\left(\frac{p}{q}\right)^{1 / q} .
\end{aligned}
$$

By using

$$
\mathcal{A}_{*}=\frac{1}{p-\alpha-1}\left(\frac{p}{q}\right)^{1 / q}
$$

on the left-hand side side of (22), we get the proof of the left-hand side of (33). On the other hand, by using (35) with $\gamma=\alpha-p+1<0$, we get

$$
\begin{aligned}
\Delta\left(k^{\alpha-p+1}\right) & =(k+1)^{\alpha-p+1}-k^{\alpha-p+1} \leq(\alpha-p+1)(k+1)^{\alpha-p}[(k+1)-k] \\
& =(\alpha-p+1)(k+1)^{\alpha-p} .
\end{aligned}
$$

Using the fact $(k+1) \leq 2 k$ for $k \geq 1$, we have that

$$
\Delta\left(k^{\alpha-p+1}\right) \leq 2^{\alpha-p}(\alpha-p+1) k^{\alpha-p},
$$


and by using (37) with $0<\gamma=\alpha(1-q)+1<1$, we obtain

$$
\begin{aligned}
\Delta\left(s^{\alpha(1-q)+1}\right) & =(s+1)^{\alpha(1-q)+1}-s^{\alpha(1-q)+1} \\
& \geq 2^{\alpha(1-q)}(\alpha(1-q)+1) s^{\alpha(1-q)} .
\end{aligned}
$$

Then from (40) and (41) in (34), we get that

$$
\begin{aligned}
& \left(\sum_{m=n}^{\infty} m^{\alpha-p}\right)^{1 / p}\left(\sum_{m=1}^{n} m^{\alpha(1-q)}\right)^{1 / q} \\
& \leq\left(\frac{\sum_{m=n}^{\infty} \Delta\left(m^{\alpha-p+1}\right)}{2^{\alpha-p}(\alpha-p+1)}\right)^{1 / p}\left(\frac{\sum_{m=1}^{n} \Delta\left(m^{\alpha(1-q)+1}\right)}{2^{\alpha(1-q)}(\alpha(1-q)+1)}\right)^{1 / q} \\
& \leq \frac{2}{p-\alpha-1}\left(\frac{p}{q}\right)^{1 / q} .
\end{aligned}
$$

By using

$$
\mathcal{A}^{*}=\frac{2}{p-\alpha-1}\left(\frac{p}{q}\right)^{1 / q}
$$

on the right-hand side of (22), we have that

$$
\|\hbar\|_{\operatorname{Ces}_{\alpha}^{p}(\mathbb{N})} \leq \frac{2 p \mathcal{S}}{(p-\alpha-1)}
$$

which leads to the proof of the left-hand side of (33). The proof is complete.

\section{Weighted Copson sequences space}

In this section, we prove a theorem of factorization of the Copson $\operatorname{space}^{\operatorname{Cop}_{\lambda}^{p}}(\mathbb{N})$ of discrete weights; as a consequence, we obtain the well-known forms of the discrete Copson type inequalities with best constants. We consider the two cases $p>1$ and $p=1$.

Definition 4.1 The discrete weighted Copson space $\operatorname{Cop}_{\lambda}^{p}(\mathbb{N})$ for $p \geq 1$ with a weight $\lambda$ by the space of all sequences $\eta$ defined on $\mathbb{N}$ is defined by

$$
\|\eta\|_{\operatorname{Cop}_{\lambda}^{p}(\mathbb{N})}=\left(\sum_{n=1}^{\infty} \lambda(n)\left(\sum_{m=n}^{\infty} \frac{|\eta(m)|}{m}\right)^{p}\right)^{1 / p}<\infty .
$$

Definition 4.2 We define the discrete weighted space $\mathcal{B}_{\lambda}^{q}(\mathbb{N})$ for $q \geq 1$ with a weight $\lambda$ by the space of all sequences $\beta$ defined on $\mathbb{N}$ such that

$$
\|\beta\|_{B_{\lambda}^{q}(\mathbb{T})}=\sup _{n>0}\left(\sum_{s=n}^{\infty} \frac{\lambda(s)}{s^{q}}\right)^{-1 / q}\left(\sum_{s=n}^{\infty} \frac{|\beta(s)|^{q} \lambda(s)}{s^{q}}\right)^{1 / q}<\infty .
$$

Claim 2 For $p \geq 1$, the space $\operatorname{Cop}_{\lambda}^{p}(\mathbb{N})$ with a norm (43) is a Banach space, and if the weight $\lambda$ satisfies (9), then $\mathcal{M}$ is bounded in $l_{\lambda}^{p}(\mathbb{N})$ and we have $l_{\lambda}^{p}(\mathbb{N}) \subseteq \operatorname{Cop}_{\lambda}^{p}(\mathbb{N})$. 
We denote

$$
\overline{\mathcal{J}}=\inf \left\{\|\eta\|_{l_{\lambda}^{p}(\mathbb{N})}\|\beta\|_{\mathcal{B}_{\lambda}^{q}(\mathbb{N})}\right\}
$$

where the infimum is taken over all possible decompositions of $\hbar=\eta \times \beta$ with $\eta \in l_{\lambda}^{p}(\mathbb{N})$ and $\beta \in \mathcal{B}_{\lambda}^{q}(\mathbb{N})$.

Definition 4.3 We denote by $\mathcal{W}^{*}$ the class of discrete weights $\lambda$ such that, for all $n \geq 1$, it follows that

$$
\left(\sum_{m=n}^{\infty} \frac{\lambda^{1-q}(m)}{m^{q}}\right)^{1 / q}\left(\sum_{m=1}^{n} \lambda(m)\right)^{1 / p} \leq \mathcal{D}^{*}
$$

for $p>1$, where $\mathcal{D}^{*}$ is the smallest constant such that (45) holds.

Definition 4.4 For $p>1$, we denote by $\mathcal{W}_{*}$ the class of discrete weights $\lambda$ such that, for all $n \geq 1$, it follows that the reverse of inequality (45) holds, and we denote by $\mathcal{D}_{*}$ the largest constant for which the reverse holds.

Definition 4.5 We say that the weight $\lambda$ belongs to $\mathcal{W}$ if it belongs to $\mathcal{W}^{*}$ and $\mathcal{W}_{*}$.

Theorem 4.1 Assume that $p>1$ and $\lambda \in \mathcal{W}$. The sequence $\hbar$ belongs to $\operatorname{Cop}_{\lambda}^{p}(\mathbb{N})$ iff it can be represented by the factorization $\hbar=\eta \times \beta$ with $\eta \in l_{\lambda}^{p}(\mathbb{N})$ and $\beta \in \mathcal{B}_{\left(\lambda^{1-q}\right)}^{q}(\mathbb{N})$. Moreover,

$$
\mathcal{D}_{*} \overline{\mathcal{J}} \leq\|\hbar\|_{\operatorname{Cop}_{\lambda}^{p}(\mathbb{N})} \leq q^{1 / q} p^{1 / p} \mathcal{D}^{*} \overline{\mathcal{J}}
$$

Proof First, we prove the imbedding $\hookrightarrow$ i.e. we prove the sequence $\hbar=\eta \times \beta \in \operatorname{Cop}_{\lambda}^{p}(\mathbb{N})$ for $\eta \in l_{\lambda}^{p}(\mathbb{N})$ and $\beta \in \mathcal{B}_{(\lambda 1-q)}^{q}(\mathbb{N})$. By applying Hölder's inequality, we see that

$$
\sum_{m=n}^{\infty} \frac{\eta(m)|\beta(m)|}{m} \leq\left(\sum_{m=n}^{\infty} \frac{\eta^{p}(m) \lambda(m)}{w^{p}(m)}\right)^{1 / p}\left(\sum_{m=n}^{\infty}|\beta(m)|^{q} \frac{\lambda^{1-q}(m)}{m^{q}} w^{q}(m)\right)^{1 / q},
$$

where $w$ is arbitrary positive, and an increasing sequence will be defined later. From (44), we see that

$$
\sum_{m=n}^{\infty}|\beta(m)|^{q} \frac{\lambda^{1-q}(m)}{m^{q}} \leq\left(\|\beta\|_{\mathcal{B}_{(\lambda-q)}^{q}}(\mathbb{N})\right)^{q} \sum_{m=n}^{\infty} \frac{\lambda^{1-q}(m)}{m^{q}} .
$$

Now, by applying Lemma 2.4 with $|\hbar(n)|=w^{q}(n)$, which is an increasing sequence, and $C=1$, we obtain that

$$
\left(\sum_{m=n}^{\infty}|\beta(m)|^{q} \frac{\lambda^{1-q}(m)}{m^{q}} w^{q}(m)\right)^{1 / q} \leq\|\beta\|_{\mathcal{B}_{(\lambda-q)}^{q}}(\mathbb{N})\left(\sum_{m=n}^{\infty} \frac{\lambda^{1-q}(m)}{m^{q}} w^{q}(m)\right)^{1 / q} .
$$


Multiplying (47) by $\lambda(n)$ and raising to the power $p$ and summing from 1 to $\infty$, we get

$$
\begin{aligned}
\sum_{n=1}^{\infty} \lambda(n)\left(\sum_{m=n}^{\infty} \frac{|\hbar(m)|}{m}\right)^{p} \leq & \|\beta\|_{\mathcal{B}_{(\lambda 1-q)}^{q}(\mathbb{N})}^{p} \sum_{n=1}^{\infty} \lambda(n)\left(\sum_{m=n}^{\infty} \frac{\eta^{p}(m) \lambda(m)}{w^{p}(m)}\right) \\
& \times\left(\sum_{m=n}^{\infty} \frac{\lambda^{1-q}(m)}{m^{q}} w^{q}(m)\right)^{p-1} .
\end{aligned}
$$

By applying Lemma 2.5, we have

$$
\begin{aligned}
\sum_{n=1}^{\infty} \lambda(n)\left(\sum_{m=n}^{\infty} \frac{|\hbar(m)|}{m}\right)^{p} \leq & \|\beta\|_{B_{\left(\lambda^{1}-q\right)}^{p}(\mathbb{N})}^{p} \sum_{n=1}^{\infty} \frac{\eta^{p}(n) \lambda(n)}{w^{p}(n)} \\
& \times\left(\sum_{\tau=1}^{n} \lambda(\tau)\left(\sum_{m=\tau}^{\infty} \frac{\lambda^{1-q}(m)}{m^{q}} w^{q}(m)\right)^{p-1}\right) .
\end{aligned}
$$

By taking $w(n)=\left(\sum_{s=n}^{\infty} \lambda^{1-q}(s) / s^{q}\right)^{-1 / p q}$ (note that $w$ is an increasing sequence) and from Lemma 2.2, we get that

$$
\begin{aligned}
\sum_{m=n}^{\infty} \frac{\lambda^{1-q}(m)}{m^{q}} w^{q}(m) & =\sum_{m=n}^{\infty} \frac{\lambda^{1-q}(m)}{m^{q}}\left(\sum_{s=m}^{\infty} \frac{\lambda^{1-q}(s)}{s^{q}}\right)^{1-\frac{1}{q}} \\
& \leq q\left(\sum_{m=n}^{\infty} \frac{\lambda^{1-q}(m)}{m^{q}}\right)^{1 / q}
\end{aligned}
$$

By combining (48) and (49), we have that

$$
\begin{aligned}
\sum_{n=1}^{\infty} \lambda(n) & \left(\sum_{m=n}^{\infty} \frac{|\hbar(m)|}{m}\right)^{p} \\
\leq & q^{p-1}\|\beta\|_{\mathcal{B}_{(\lambda 1-q)}^{q}(\mathbb{N})}^{p} \sum_{n=1}^{\infty} \eta^{p}(n) \lambda(n)\left(\sum_{s=n}^{\infty} \frac{\lambda^{1-q}(s)}{s^{q}}\right)^{1 / q} \\
& \times\left(\sum_{\tau=1}^{n} \lambda(\tau)\left(\sum_{m=\tau}^{\infty} \frac{\lambda^{1-q}(m)}{m^{q}}\right)^{(p-1) / q}\right) \\
= & q^{p-1}\|\beta\|_{\mathcal{B}_{(\lambda)}^{q}}^{p}\left(\sum_{n=1}^{\infty} \sum_{n=1}^{\infty} \eta^{p}(n) \lambda(n)\left(\sum_{s=n}^{\infty} \frac{\lambda^{1-q}(s)}{s^{q}}\right)^{1 / q}\right. \\
& \times \sum_{\tau=1}^{n} \lambda(\tau)\left(\sum_{m=1}^{n} \lambda(m)\right)^{\frac{1}{p}-1}\left(\sum_{m=n}^{\infty} \frac{\lambda^{1-q}(m)}{m^{q}}\right)^{(p-1) / q}\left(\sum_{m=1}^{n} \lambda(m)\right)^{\frac{p-1}{p}} .
\end{aligned}
$$

By the definition of $\mathcal{D}^{*}$, we get

$$
\begin{aligned}
\sum_{n=1}^{\infty} \lambda(n)\left(\sum_{m=n}^{\infty} \frac{|\hbar(m)|}{m}\right)^{p} \leq & q^{p-1}\left(\mathcal{D}^{*}\right)^{p-1}\|\beta\|_{\mathcal{W}_{(\lambda 1-q)}^{q}}^{p}(\mathbb{N}) \sum_{n=1}^{\infty} \eta^{p}(n) \lambda(n) \\
& \times\left(\sum_{s=n}^{\infty} \frac{\lambda^{1-q}(s)}{s^{q}}\right)^{1 / q} \sum_{\tau=1}^{n} \lambda(\tau)\left(\sum_{m=1}^{n} \lambda(m)\right)^{\frac{1}{p}-1} .
\end{aligned}
$$


Again by applying (10) with $\phi=\lambda$ and $\gamma=1 / p$, we have that

$$
\begin{aligned}
\sum_{n=1}^{\infty} \lambda(n)\left(\sum_{m=n}^{\infty} \frac{|\hbar(m)|}{m}\right)^{p} \leq & p q^{p-1}\left(\mathcal{D}^{*}\right)^{p-1}\|\beta\|_{\mathcal{B}_{(\lambda 1-q)}^{q}}^{p}(\mathbb{N}) \\
& \times\left(\sum_{n=1}^{\infty} \frac{\lambda^{1-q}(s)}{s^{q}}\right)^{p}\left(\eta_{m=1}^{1 / q}(n) \lambda(n)\right. \\
\leq & p q^{p-1}\left(\mathcal{D}^{*}\right)^{p}\|\beta\|_{\mathcal{B}_{(\lambda 1-q)}^{q}(\mathbb{N})}^{p} \sum_{n=1}^{1 / p} \eta^{p}(n) \lambda(n) \\
= & p q^{p-1}\left(\mathcal{D}^{*}\right)^{p}\|\beta\|_{\mathcal{B}_{(\lambda)}^{q}(-q)}^{p}(\mathbb{N})
\end{aligned}
$$

This implies that $\hbar \in \operatorname{Cop}_{\lambda}^{p}(\mathbb{N})$, and

$$
\|\hbar\|_{\operatorname{Cop}_{\lambda}^{p}(\mathbb{N})} \leq q^{1 / q} p^{1 / p} \mathcal{D}^{*} \inf \left\{\|\beta\|_{\mathcal{B}_{(\lambda-q)}^{q}(\mathbb{N})}\|\eta\|_{l_{\lambda}^{p}(\mathbb{N})}\right\}
$$

which proves the first part. For the reversed direction $\hookleftarrow$, we will prove that $\operatorname{Cop}_{\lambda}^{p}(\mathbb{N}) \subset$ $l_{\lambda}^{p}(\mathbb{N}) \cdot \mathcal{B}_{\left(\lambda^{1-q}\right)}^{q}(\mathbb{N})$. Let $\hbar \in \operatorname{Cop}_{\lambda}^{p}(\mathbb{N})$ and assume that

$$
v(n)=\frac{1}{n \lambda(n)} \sum_{m=1}^{n} \lambda(m)\left(\sum_{s=m}^{\infty} \frac{\hbar(s)}{s}\right)^{p-1}>0 .
$$

Define

$$
\eta(n)=|\hbar|^{1 / p}(n) v^{1 / p}(n) \operatorname{sign} \hbar(n), \quad \text { and } \quad \beta(n)=|\hbar|^{1 / q}(n) v^{-1 / p}(n)
$$

As in the proof of Theorem 3.1, we can see that $\|\eta\|_{l_{\lambda}^{p}(\mathbb{N})}=\|\hbar\|_{\operatorname{Cop}_{\lambda}^{p}(\mathbb{N})}<\infty($ see (27)). By using the definition of $\beta$ and applying Hölder's inequality, we have

$$
\left(\sum_{s=n}^{\infty}|\beta(s)|^{q} \frac{\lambda^{1-q}(s)}{s^{q}}\right)^{p} \leq\left(\sum_{s=n}^{\infty} \frac{|\hbar(s)|}{s}\right)^{p-1}\left(\sum_{s=n}^{\infty} \frac{|\hbar(s)|}{s} v^{-q}(s) \frac{\lambda^{-q}(s)}{s^{q}}\right)
$$

By multiplying inequality (51) by $\sum_{s=1}^{n} \lambda(s)$ and using the fact that $n \lambda(n) \nu(n)$ is increasing (see (50)), we get that

$$
\begin{aligned}
& \left(\sum_{s=1}^{n} \lambda(s)\right)\left(\sum_{s=n}^{\infty}|\beta(s)|^{q} \frac{\lambda^{1-q}(s)}{s^{q}}\right)^{p} \\
& \quad \leq\left(\sum_{s=1}^{n} \lambda(s)\right)\left(\sum_{s=n}^{\infty} \frac{|\hbar(s)|}{s}\right)^{p-1}\left(\sum_{s=n}^{\infty} \frac{|\hbar(s)|}{s} v^{-q}(s) \frac{\lambda^{-q}(s)}{s^{q}}\right) \\
& \leq\left(\sum_{s=1}^{n} \lambda(s)\left(\sum_{m=s}^{\infty} \frac{|\hbar(m)|}{m}\right)^{p-1}\right)\left(\sum_{s=n}^{\infty} \frac{|\hbar(s)|}{s} v^{-q}(s) \frac{\lambda^{-q}(s)}{s^{q}}\right)
\end{aligned}
$$




$$
\begin{aligned}
& =n v(n) \lambda(n)\left(\sum_{s=n}^{\infty} \frac{|\hbar(s)|}{s} v^{-q}(s) \frac{\lambda^{-q}(s)}{s^{q}}\right) \\
& \leq\left(\sum_{s=n}^{\infty}|\hbar(s)| v^{1-q}(s) \frac{\lambda^{1-q}(s)}{s^{q}}\right)=\sum_{s=n}^{\infty}|\beta(s)|^{q} \frac{\lambda^{1-q}(s)}{s^{q}} .
\end{aligned}
$$

Since $\beta^{q}(s)=|\hbar(s)| v^{1-q}(s)$, we obtain

$$
\left(\sum_{s=n}^{\infty} \frac{\lambda^{1-q}(s)}{s^{q}}\right)^{-1 / q}\left(\sum_{s=n}^{\infty} \beta^{q}(s) \frac{\lambda^{1-q}(s)}{s^{q}}\right)^{1 / q} \leq\left(\sum_{s=n}^{\infty} \frac{\lambda^{1-q}(s)}{s^{q}}\right)^{-1 / q}\left(\sum_{s=1}^{n} \lambda(s)\right)^{-1 / p} .
$$

Hence

$$
\left(\sum_{s=n}^{\infty} \frac{\lambda^{1-q}(s)}{s^{q}}\right)^{-1 / q}\left(\sum_{s=n}^{\infty}|\beta(s)|^{q} \frac{\lambda^{1-q}(s)}{s^{q}}\right)^{1 / q} \leq \frac{1}{\mathcal{D}_{*}}
$$

which means that $\beta$ belongs to $\mathcal{B}_{\left(\lambda^{1-q}\right)}^{q}(\mathbb{N})$. Moreover, we have that

$$
\|\hbar\|_{\operatorname{Cop}_{\lambda}^{p}(\mathbb{N})}=\|\eta\|_{l_{\lambda}^{p}(\mathbb{N})} \geq \mathcal{D}_{*} \inf \|\eta\|_{l_{\lambda}^{p}(\mathbb{N})}\|\beta\|_{\mathcal{B}_{(\lambda 1-q)}^{q}(\mathbb{N})},
$$

which leads to the proof of the second part. The proof is complete.

Example 2 If we take $\beta(n)=1$, then inequality (46) gives the boundedness of the weighted Copson operator for $1<p<\infty$ on the space $l_{\lambda}^{p}(\mathbb{N})$.

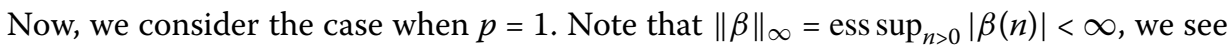
that

$$
\sum_{s=n}^{\infty} \frac{|\beta(s)|^{p} \lambda(s)}{s^{p}} \leq\|\beta\|_{\infty} \sum_{s=n}^{\infty} \frac{\lambda(s)}{s^{p}}
$$

so we are able to replace the space $\mathcal{B}_{\lambda}^{1}(\mathbb{N})$ with the space $l^{\infty}(\mathbb{N})$. Now, we consider the space $\mathcal{V}$ of weights $\lambda$ such that

$$
\frac{1}{n} \sum_{m=1}^{n} \lambda(m) \leq C \lambda(n)
$$

and assume that there exist a positive constant $\mathcal{V}^{*}$, the smallest constant for which inequality (52) holds, and a positive constant $\mathcal{V}_{*}$, which is the largest constant for which the reverse of it holds. In the following, we denote

$$
\overline{\mathcal{F}}=\inf \left\{\|\eta\|_{l_{\lambda}^{1}(\mathbb{N})}\|\beta\|_{\infty}\right\}
$$

where the infimum is taken over all possible decompositions of $\hbar=\eta \times \beta$ with $\eta \in l_{\lambda}^{1}(\mathbb{N})$ and $\beta \in l^{\infty}(\mathbb{N})$. 
The proof of the following theorem is similar to the proof of Theorem 3.2 by using the class of the sequences $\mathcal{V}$ and the sequence

$$
v(n)=\frac{1}{n \lambda(n)} \sum_{m=1}^{n} \lambda(m)>0
$$

and hence it is omitted.

Theorem 4.2 Let $\lambda$ belong to the class $\mathcal{V}$. The sequence $\hbar \in \operatorname{Cop}_{\lambda}^{1}(\mathbb{N})$ if and only if it admits a factorization $\hbar=\eta \times \beta$ with $\eta \in l_{\lambda}^{1}(\mathbb{N})$ and $\beta \in l^{\infty}(\mathbb{N})$. Moreover,

$$
\mathcal{V}_{*} \overline{\mathcal{F}} \leq\|\hbar\|_{\operatorname{Cop}_{\lambda}^{1}(\mathbb{N})} \leq \mathcal{V}^{*} \overline{\mathcal{F}}
$$

Remark 4.1 This theorem proves the sufficient condition of the boundedness of adjoint Hardy's operator $\mathcal{M} \eta(n):=\sum_{s=n}^{\infty}(|\eta(s)| / s)$ for $n>0$ on $l_{\lambda}^{1}(\mathbb{N})$.

In the following, we will denote by $l_{n^{\alpha}}^{p}(\mathbb{N}), \mathcal{B}_{n^{\alpha(1-q)}}^{q}(\mathbb{N})$, and $\operatorname{Cop}_{n^{\alpha}}^{p}(\mathbb{N})$ the spaces of power weights $\lambda(n)=n^{\alpha}$. In analogy with the general case, we also denote

$$
\overline{\mathcal{S}}:=\inf \left\{\|\eta\|_{l_{n^{\alpha}}^{p}(\mathbb{N})}\|\beta\|_{\mathcal{B}_{n^{\alpha(1-q)}}^{q}(\mathbb{N})}\right\}
$$

with $\eta \in l_{n^{\alpha}}^{p}(\mathbb{N})$ and $\beta \in \mathcal{B}_{n^{\alpha(1-q)}}^{q}(\mathbb{N})$, and the infimum is taken over all possible decompositions of $\hbar=\eta \times \beta$. This gives us the following result.

Corollary 4.1 Assume that $p>1$ and $p-1>\alpha>0$. Then $\hbar \in \operatorname{Cop}_{n^{\alpha}}^{p}(\mathbb{N})$ iff it admits a factorization $\hbar=\eta \times \beta$ with $\eta \in l_{n^{\alpha}}^{p}(\mathbb{N})$ and $\beta \in \mathcal{B}_{n^{\alpha(1-q)}}^{q}(\mathbb{N})$. Moreover,

$$
2^{\frac{-\alpha}{p}} \frac{(p-1)^{1 / q} \overline{\mathcal{S}}}{(\alpha+1)} \leq\left(\sum_{n=1}^{\infty}\left(\sum_{m=n}^{\infty} \frac{|\eta(m)|}{m}\right)^{p} n^{\alpha}\right)^{1 / p} \leq 2^{\frac{\alpha}{p}+1} \frac{p \overline{\mathcal{S}}}{(\alpha+1)}
$$

Proof By using $\lambda(n)=n^{\alpha}$, we see that the condition in (45) now reads

$$
\left(\sum_{m=1}^{n} m^{\alpha}\right)^{1 / p}\left(\sum_{m=n}^{\infty} m^{\alpha(1-q)-q}\right)^{1 / q}
$$

By employing inequality (35) with $\beta=\alpha+1>1$, we obtain

$$
\begin{aligned}
\Delta\left(s^{\alpha+1}\right) & =(s+1)^{\alpha+1}-s^{\alpha+1} \leq(\alpha+1)(s+1)^{\alpha}((s+1)-s) \\
& =(\alpha+1)(s+1)^{\alpha} .
\end{aligned}
$$

By using $(m+1)<2 m$, we get

$$
\Delta\left(s^{\alpha+1}\right) \leq 2^{\alpha}(\alpha+1) s^{\alpha} .
$$


For $\beta=\alpha(1-q)-q+1<0$, we also have

$$
\begin{aligned}
\Delta\left(s^{\alpha(1-q)-q+1}\right) & =(s+1)^{\alpha(1-q)-q+1}-s^{\alpha(1-q)-q+1} \\
& \geq(\alpha(1-q)-q+1) s^{\alpha(1-q)-q}((s+1)-s) \\
& =(\alpha(1-q)-q+1) s^{\alpha(1-q)-q} .
\end{aligned}
$$

Then from (55) and (56) in (54) we get

$$
\begin{aligned}
& \left(\sum_{m=1}^{n} m^{\alpha}\right)^{1 / p}\left(\sum_{m=n}^{\infty} m^{\alpha(1-q)-q}\right)^{1 / q} \\
& \geq\left(\frac{\sum_{m=1}^{n} \Delta\left(m^{\alpha+1}\right)}{2^{\alpha}(\alpha+1)}\right)^{1 / p}\left(\frac{\sum_{m=n}^{\infty} \Delta\left(m^{\alpha(1-q)-q+1}\right)}{(\alpha(1-q)-q+1)}\right)^{1 / q} \\
& =2^{\frac{-\alpha}{p}}\left(\frac{n^{(\alpha+1) / p}}{(\alpha+1)^{1 / p}}\right)\left(\frac{-n^{\frac{\alpha}{q}(1-q)-\frac{1}{p}}}{(\alpha(1-q)-q+1)^{1 / q}}\right) \\
& =\frac{-2^{\frac{-\alpha}{p}}}{\alpha+1}(-p / q)^{1 / q}=2^{\frac{-\alpha}{p}} \frac{(p-1)^{1 / q}}{\alpha+1} .
\end{aligned}
$$

By using

$$
\mathcal{D}_{*}=2^{\frac{-\alpha}{p}} \frac{(p-1)^{1 / q}}{\alpha+1},
$$

on the left-hand side of (46), we get the proof of the left-hand side of (53). On the other hand, by using (35) with $\gamma=\alpha+1>1$, we obtain

$$
\begin{aligned}
\Delta\left(s^{\alpha+1}\right) & =(s+1)^{\alpha+1}-s^{\alpha+1} \geq(\alpha+1) s^{\alpha}((s+1)-s) \\
& =(\alpha+1) s^{\alpha} .
\end{aligned}
$$

For $\gamma=\alpha(1-q)-q+1<0$, we also have

$$
\begin{aligned}
\Delta\left(s^{\alpha(1-q)-q+1}\right) & =(s+1)^{\alpha(1-q)-q+1}-s^{\alpha(1-q)-q+1} \\
& \leq(\alpha(1-q)-q+1)(s+1)^{\alpha(1-q)-q}((s+1)-s) \\
& =2^{\alpha(1-q)-q}(\alpha(1-q)-q+1) s^{\alpha(1-q)-q} .
\end{aligned}
$$

From (57) and (58) in (54) we get

$$
\begin{aligned}
& \left(\sum_{m=1}^{n} m^{\alpha}\right)^{1 / p}\left(\sum_{m=n}^{\infty} m^{\alpha(1-q)-q}\right)^{1 / q} \\
& \leq\left(\frac{\sum_{m=1}^{n} \Delta\left(m^{\alpha+1}\right)}{(\alpha+1)}\right)^{1 / p}\left(\frac{\sum_{m=n}^{\infty} \Delta\left(m^{\alpha(1-q)-q+1}\right)}{2^{\alpha(1-q)-q}(\alpha(1-q)-q+1)}\right)^{1 / q} \\
& \quad=2^{\frac{\alpha(q-1)+q}{q}}\left(\frac{n^{(\alpha+1) / p}}{(\alpha+1)^{1 / p}}\right)\left(\frac{-n^{\frac{\alpha}{q}(1-q)-\frac{1}{p}}}{(\alpha(1-q)-q+1)^{1 / q}}\right) \\
& \leq \frac{-2^{\frac{\alpha}{p}+1}}{(\alpha+1)(1-q)^{1 / q}}=2^{\frac{\alpha}{p}+1} \frac{(p-1)^{1 / q}}{\alpha+1}
\end{aligned}
$$


So from (46) and using

$$
\mathcal{D}^{*}=2^{\frac{\alpha}{p}+1} \frac{(p-1)^{1 / q}}{\alpha+1}
$$

we have that

$$
\|h\|_{\operatorname{Cop}_{\alpha}^{p}(\mathbb{T})} \leq 2^{\frac{\alpha}{p}+1} q^{1 / q} p^{1 / p} \frac{(p-1)^{1 / q}}{\alpha+1}=2^{\frac{\alpha}{p}+1} \frac{p}{\alpha+1},
$$

which leads to the proof of the right-hand side of (53). The proof is complete.

Remark 4.2 In Corollary 4.1, if $\alpha=0$, then we get the result that has been obtained in [8].

Conclusion In this paper the authors studied the discrete weighed Cesàro and Copson sequence spaces. They derived and proved some properties of these spaces by establishing some factorization theorems and also obtained conditions for the boundedness of the generalized discrete Hardy and Copson operators.

\section{Acknowledgements}

The third author extend his appreciation to the Deanship of Scientific Research at King Khalid University for funding this work through General Research Project under Grant No. G.R.P-172-41. The authors thank the referees for their comments that led to the improvement of the presentation of the results in this paper.

\section{Funding}

The third author was supported by the project under grant number G.R.P.1-162-40 from King Khalid University.

\section{Availability of data and materials}

Not applicable.

\section{Competing interests}

The authors declare that they have no competing interests.

\section{Authors' contributions}

All authors contributed equally to the writing of this paper. All authors read and approved the final manuscript.

\section{Author details}

${ }^{1}$ Mathematics Division, Faculty of Basic Science, Galala University, Galala New City 43511, Egypt. ${ }^{2}$ Department of Mathematics, Faculty of Science, Mansoura University, Mansoura 35516, Egypt. ${ }^{3}$ Department of Mathematics, College of Science, King Khalid University, 9004 Abha, Saudi Arabia. ${ }^{4}$ Department of Mathematics, Faculty of Science, Al-Azhar University, Z 71524 Assiut, Egypt. ${ }^{5}$ Department of Mathematics, Cankaya University, Ankara, Turkey. ${ }^{6}$ Institute of Space Sciences, Magurele-Bucharest, Romania.

\section{Publisher's Note}

Springer Nature remains neutral with regard to jurisdictional claims in published maps and institutional affiliations.

Received: 11 November 2019 Accepted: 4 December 2020 Published online: 04 January 2021

\section{References}

1. Agarwal, R.P., Mahmoud, R.R., Saker, S.H., Tunć, C.: New generalizations of Németh-Mohapatra type inequalities on time scales. Acta Math. Hung. 152, 383-403 (2017)

2. Anderson, K.F., Heinig, H.P.: Weighted norm inequalities for certain integral operators. SIAM J. Math. Anal. 14, 834-844 (1983)

3. Arińo, M.A., Muckenhoupt, B.: A characterization of the dual of the classical Lorentz sequence space $d(w, q)$. Proc. Am. Math. Soc. 112, 87-89 (1991)

4. Astashkin, S., Maligranda, L.: Cesàro function spaces fail to have a fixed point property. Proc. Am. Math. Soc. 136, 4289-4294 (2008)

5. Astashkin, S., Maligranda, L.: Structure of Cesàro function spaces. Indag. Math. 20(3), 329-379 (2009)

6. Bala, I.: On Cesàro sequence space defined by a modulus function. Demonstr. Math. XLVI, 157-163 (2013)

7. Barza, S., Marcoci, A., Marcoci, L.: Factorizations of weighted Hardy inequalities. Bull. Braz. Math. Soc. 49(4), 915-932 (2018)

8. Bennett, G.: Factorizing the classical inequalities. Mem. Am. Math. Soc. 120(576), 1-130 (1996) 
9. Bennett, G., Grosse-Erdmann, K.-G.: On series of positive terms. Houst. J. Math. 31, 541-586 (2005)

10. Carton-Lebrun, C., Heinig, H.P.: Weight characterization of an averaging operator. J. Math. Anal. Appl. 283(1), 236-243 (2003)

11. Copson, E.T: Note on series of positive terms. J. Lond. Math. Soc. 2, 9-12 (1927)

12. Copson, E.T.: Note on series of positive terms. J. Lond. Math. Soc. 3, 49-51 (1928)

13. Hardy, G.H.: Notes on a theorem of Hilbert. Math. Z. 6, 314-317 (1920)

14. Hardy, G.H., Littlewood, J.E., Polya, G.: Inequalities, 2nd edn. Cambridge University Press, Cambridge (1934)

15. Hassard, B.D., Hussein, D.A.: On Cesàro function spaces. Tamkang J. Math. 4, 19-25 (1973)

16. Jagers, A.A.: A note on Cesàro sequence spaces. Nieuw Arch. Wiskd. (3) 22, 113-124 (1974)

17. Johnson, P.D. Jr., Mohapatra, R.N.: Density of finitely non-zero sequences in some sequence spaces. Math. Jpn. 24 253-262 (1979)

18. Johnson, P.D. Jr., Mohapatra, R.N.: On inequalities related to sequence spaces ces[p, q]. In: Walter, W. (ed.) General Inequalities 4. International Series of Numerical Mathematics, vol. 71, pp. 191-201. Birkhäuser, Basel (1984)

19. Johnson, P.D. Jr., Mohapatra, R.N.: On an analogue of Hardy's inequality. Arch. Math. 60, 157-163 (1993)

20. Leibowitz, G.M.: A note on the Cesàro sequence spaces. Tamkang J. Math. 2, 151-157 (1971)

21. Leindler, L.: An addition to factorization of inequalities. Math. Pannon. 10, 61-65 (1999)

22. Leśnik, K., Maligranda, L.: Abstract Cesàro spaces. Duality J. Math. Anal. Appl. 424, 932-951 (2015)

23. Manna, A.: Factorized enhancement of Copson's inequality. Tamkang J. Math. 49, 195-203 (2018)

24. Opic, B., Kufner, A.: Hardy-Type Inequalities. Pitman Research Notes in Mathematics Series. Longman, Harlow (1990)

25. Saker, S.H., Tunć, C., Mahmoud, R.: New Carlson-Bellman and Hardy-Littlewood dynamic inequalities. Math. Inequal. Appl. 21, 967-983 (2018)

26. Shiue, J.S.: On the Cesàro sequence spaces. Tamkang J. Math. 1, 19-25 (1970)

27. Suantai, S.: On some convexity properties of generalized Cesàro sequence spaces. Georgian Math. J. 10, 193-200 (2003)

28. Sy, P.W., Zhang, W.Y., Lee, P.Y.: The dual of Cesàro function spaces. Glas. Mat. Ser. III 22, 103-112 (1987)

29. Yee, L.P.: Cesàro sequence spaces. Math. Chron. 13, 29-45 (1984)

\section{Submit your manuscript to a SpringerOpen ${ }^{\circ}$ journal and benefit from:}

- Convenient online submission

- Rigorous peer review

- Open access: articles freely available online

- High visibility within the field

- Retaining the copyright to your article

Submit your next manuscript at $\gg$ springeropen.com 